\title{
Benchmarking life expectancy and cancer mortality: global comparison with cardiovascular disease 1981-2010
}

\author{
OPEN ACCESS
}

\author{
Bochen Cao postdoctoral research fellow ${ }^{1}$, Freddie Bray scientist ${ }^{1}$, Hiram Beltrán-Sánchez assistant \\ professor $^{2}$, Ophira Ginsburg associate professor ${ }^{3}$, Samir Soneji assistant professor ${ }^{4}$, Isabelle \\ Soerjomataram scientist ${ }^{1}$
}

${ }^{1}$ Section of Cancer Surveillance, International Agency for Research on Cancer, 69372 Lyon CEDEX 08, France; ${ }^{2}$ Fielding School of Public Health and California Center for Population Research, University of California, Los Angeles, CA, USA; ${ }^{2}$ Laura and Isaac Perlmutter Cancer Center, Department of Population Health, NYU Langone Medical Center, New York, NY, USA; ${ }^{4}$ Dartmouth Institute for Health Policy and Clinical Practice, Geisel School of Medicine, Dartmouth College, Hanover, NH, USA

\begin{abstract}
Objective To quantify the impact of cancer (all cancers combined and major sites) compared with cardiovascular disease (CVD) on longevity worldwide during 1981-2010.

Design Retrospective demographic analysis using aggregated data.

Setting National civil registration systems in member states of the World Health Organization.

Participants 52 populations with moderate to high quality data on cause specific mortality.

Main outcome measures Disease specific contributions to changes in life expectancy in ages $40-84\left(\mathrm{LE}_{40-84}\right)$ over time in populations grouped by two levels of Human Development Index (HDI) values.

Results Declining CVD mortality rates during 1981-2010 contributed to, on average, over half of the gains in $\mathrm{LE}_{40-84}$; the corresponding gains were 2.3 (men) and 1.7 (women) years, and 0.5 (men) and 0.8 (women) years in very high and medium and high HDI populations, respectively. Declines in cancer mortality rates contributed to, on average, $20 \%$ of the gains in $\mathrm{LE}_{40-84}$, or 0.8 (men) and 0.5 (women) years in very high $\mathrm{HDI}$ populations, and to over $10 \%$ or 0.2 years (both sexes) in medium and high HDI populations. Declining lung cancer mortality rates brought about the largest $\mathrm{LE}_{40-84}$ gain in men in very high $\mathrm{HDI}$ populations (up to 0.7 years in the Netherlands), whereas in medium and high HDI populations its contribution was smaller yet still positive. Among women, declines in breast cancer mortality rates were largely responsible for the improvement in longevity, particularly among very high HDI populations (up to 0.3 years in the United Kingdom). In contrast, losses in $L_{40-84}$ were observed in many medium and high HDI populations as a result of increasing breast cancer mortality rates.
\end{abstract}

Conclusions The control of CVD has led to substantial gains in $\mathrm{LE}_{40-84}$ worldwide. The inequality in improvement in longevity attributed to declining cancer mortality rates reflects inequities in implementation of cancer control, particularly in less resourced populations and in women. Global actions are needed to revitalize efforts for cancer control, with a specific focus on less resourced countries.

\section{Introduction}

Cancer is surpassing cardiovascular disease (CVD) as the leading cause of death in many high income populations and is projected to become a leading cause of morbidity and mortality worldwide in the coming decades. ${ }^{1-3}$ In 2012 there were 8.2 million deaths from cancer worldwide, and in 2035 such deaths are predicted to rise to 14.6 million. ${ }^{4}$ The increasing burden is the synergistic result of ongoing demographic and epidemiological transitions. The current demographic transition is predominately characterized by population growth and aging in developing countries, ${ }^{5}$ leading to an increased exposure to aging related chronic diseases, including cancer. The present phase of epidemiological transition is mainly driven by the change in the cause composition of mortality and morbidity, with the disease burden shifting from infectious diseases to cancer and other major non-communicable diseases, including CVD, diabetes, and chronic respiratory diseases. ${ }^{67}$ While the substantial progress in reducing mortality from CVD has been shown, equivalent global assessment of cancer remains challenging, requiring a multifaceted and multi-indicator approach. 
Patterns and trends in cancer mortality vary noticeably between countries and across specific cancer types, reflecting the complexity of cancer, including local exposures to known or putative determinants, changing lifestyles, and the built environment. The patterns and trends also link to inherent disparities and widening gaps between and within countries in levels of health infrastructure and medical practice. ${ }^{2-12}$ Cancer mortality rates are declining in most highly developed countries, largely due to recent successes in the control of common cancers through programs of effective prevention, early detection, and treatment. In contrast, mortality rates of many types of cancer, including breast cancer and prostate cancer, are still increasing in transitioning countries, or at best stabilizing. ${ }^{8}{ }^{12}$

These diverse and complex trends in cancer mortality rates have hindered a complete understanding of the global progress in cancer control in absolute or relative terms, particularly against the backdrop of noticeable reductions in mortality rates from infectious diseases and CVD worldwide. Overwhelmingly there is a need to quantify and better understand the position of cancer among other leading causes of avoidable death, including CVD, and the specific impact of major cancers as barriers to attaining old age. In this study, we quantify the contributions of changing cancer mortality rates on changes in life expectancy in ages 40-84 ( $\left.\mathrm{LE}_{40-84}\right)$ over the period 1981-2010, adjusting for other causes of deaths, while making benchmark comparisons with equivalent gains achieved through the reduction of CVD mortality rates. Owing to the exclusive availability of mortality data in higher income countries, we include only populations indexed at relatively high levels of development. We do, however, discuss the implications of our findings beyond these settings to low and middle income countries (as classified by the World Bank based on gross national income per capita), ${ }^{13}$ which at the present time are often constrained by limited resources and capacity for cancer control.

\section{Data sources and methods}

\section{Data sources}

We extracted the total number of deaths from all causes, CVD (international classification of diseases, 10th revision (ICD-10 code I00-99)), and cancer by five year age group, sex, and year of death for the period 1981 to 2010 from the World Health Organization mortality database. ${ }^{14}$ The corresponding mid-year population counts were derived from the United Nations World Population Prospects (2015). ${ }^{15}$ For cancer deaths, we included all cancers combined (ICD-10: C00-97, B21) as well as the five most common cancer deaths: lung (C33-34), colorectum (C18-21), stomach (C16), prostate (C61), and female breast (C50). The analysis was restricted to a total of 52 populations, with cause specific and moderate to high quality mortality data according to the WHO's assessment of population coverage, completeness, and accuracy of cause of death reports. ${ }^{16}$ To ensure the comparability of cause specific mortality estimates over time, we redistributed the deaths under ICD codes indicating "symptoms, signs, and ill-defined conditions" to specific causes of death. Specifically, cancers with ill defined conditions were redistributed proportionally to all sites, excluding liver, pancreas, ovary, and lung, which do not have substantial ill defined deaths. ${ }^{17}$ Similarly, ill defined cardiovascular causes were redistributed pro-rata to ischemic heart diseases and other cardiovascular causes of death. ${ }^{17}{ }^{18}$ See supplemental table $\mathrm{S} 1$ for a list of these populations, data availability, and indicators of data quality (eg, completeness, coverage, and percentage of ill defined deaths). Also shown, in supplemental table $\mathrm{S} 2$, are key mortality indicators (eg, $\mathrm{LE}_{40-84}$ and age standardized mortality rates in 1981-85 and 2006-10) for each population.

We categorized populations based on the Human Development Index (HDI) in 2010, as reported by the United Nations Development Programme (UNDP) ${ }^{19}$ We portioned populations into two groups: populations with a HDI value above 0.80 , corresponding to very high human development by UNDP's classification, and referred to as very high HDI, and populations with a value of 0.79 or lower (the population with the lowest HDI in this analysis had a value of 0.61 ), corresponding to and referred to as medium and high HDI. ${ }^{19}$ The index provides a measurement of national wellbeing, a social indicator that considers education, adult literacy, years of schooling, healthcare (life expectancy at birth), as well as economic wealth (GDP). Although life expectancy at birth is one component of HDI and is correlated with the life expectancy measure used in this analysis $\left(\mathrm{LE}_{40-84}\right)$, exclusion of this component of HDI only marginally affected the binary stratification used in this analysis (see supplemental file).

\section{Analytical strategies}

The supplemental file provides details of the methodological steps. We performed all analyses for the five year periods in 1981-85 and in 2006-10, using the five year average of cause specific mortality rates. Briefly, we first calculated $\mathrm{LE}_{40-84}$ by population and sex using conventional demographic life table methods. ${ }^{20}$ Only ages $40-84$ were included, as individuals within this age group comprise the majority of cancer cases. In addition, in this age group there is also a lower probability of the causes of death being misreported and the existence of comorbid conditions compared with in those aged 85 or more, reducing bias related to competing causes of deaths. As a sensitivity test, we replicated the analysis for ages 0-39 and found that the contributions of cancer to change in life expectancy are negligible in this age group. Accordingly, $\mathrm{LE}_{40-84}$ throughout this article refers to life expectancy in ages $40-84$, the expected number of years lived between ages 40 and 84 , whereas we truncated years lived above age 85 . We then estimated the contribution of cancers, CVD, and all other causes of deaths to the change in $\mathrm{LE}_{40-84}$ between 1981-85 and 2006-10 using methods developed by Beltrán-Sánchez et al. ${ }^{21} 22$

\section{Patient involvement}

No patients were involved in setting the research question or the outcome measures, nor were they involved in developing plans for design or implementation of the study. No patients were asked to advise on interpretation or writing up of results. There are no plans to disseminate the results of the research to study participants or the relevant patient community.

\section{Results}

\section{Changes in mortality rates and $\mathrm{LE}_{40-84}$ in 1981-2010}

An overall decrease in mortality rates from all causes led to a noticeable increase in $\mathrm{LE}_{40-84}$ over the study period (table $1 \Downarrow$, and figure $1 \Downarrow$ ). In particular, very high HDI populations experienced gains of on average 3.7 and 2.5 years in $\mathrm{LE}_{40-84}$ among men and women, respectively, while respective values for medium and high HDI populations were lower, at 1.1 and 1.4 years (table $1 \Downarrow$ ). Decline in mortality rates from CVD was the main contributor, accounting for an average of more than $60 \%$ and $50 \%$ of declines in overall mortality rates in very high and medium and high HDI populations, respectively (table $2 \Downarrow$ ). 
Although decreasing overall cancer mortality rates were observed, they were greater in very high HDI populations (declines of $20 \%$ and $15 \%$ over the 30 year period for men and women, respectively) compared with medium and high HDI populations (4\% and 5\% decreases, respectively).

Trends by cancer types revealed heterogeneity by HDI and sex. Lung cancer mortality rates in men dropped by $26 \%$ in very high HDI populations compared with $13 \%$ in medium and high HDI populations. Conversely, lung cancer mortality rates in women increased more rapidly in very high HDI populations (40\%) than in medium and high HDI populations (12\%).

Stomach cancer alone exhibited a decline in mortality rates in both sexes during $1981-2010$, of nearly $60 \%$ and $45 \%$ in very high and medium HDI populations and in high HDI populations, respectively.

\section{Changes in $\mathrm{LE}_{40-84}$ in 1981-2010 by major causes of death}

The decline in CVD mortality rates contributed to, on average, more than half of the gain in $\mathrm{LE}_{40-84}$ in very high as well as in medium and high HDI populations (table $3 \Downarrow$ ). Declines in cancer mortality rates contributed about $20 \%$ (on average 0.8 and 0.5 years) of life years gained in ages 40-84 in men and women, respectively, in very high HDI populations, compared with $14 \%$ for men and $11 \%$ for women (a 0.2-year gain in both sexes) in medium and high HDI populations.

The largest gains in $\mathrm{LE}_{40-84}$ were observed in very high HDI populations, including Singapore, the Republic of Korea, and Chile (fig $2 \Downarrow$ ). Likewise, the changes in $\mathrm{LE}_{40-84}$ attributed to cancers were largest in the very high HDI populations, with the biggest gain observed in Singapore (1.6 and 1.0 years in men and women, respectively). Only in Lithuania and Hungary were there losses within very high HDI populations. In the medium and high HDI populations, the largest losses in $\mathrm{LE}_{40-84}$ due to cancer were seen in Romania ( 0.4 years in men) and El Salvador (0.4 years in women). Causes other than CVD and cancer contributed a greater proportion of changing $\mathrm{LE}_{40-84}$ in medium and high HDI populations compared with the highest HDI group.

\section{Changes in $\mathrm{LE}_{40-84}$ in 1981-2010 according to specific cancer sites}

Declines in lung cancer mortality rates had the largest impact on $\mathrm{LE}_{40-84}$ among men in very high $\mathrm{HDI}$ populations, contributing a gain of 0.3 years, contrasting with a slight loss ( 0.1 years) among women owing to opposing, increasing trends (table $3 \Downarrow$ ). The Netherlands and the United Kingdom showed the largest gains in $\mathrm{LE}_{40-84}$ among men as a result of declining rates, with 0.7 and 0.6 years gained, respectively (fig $3 \Downarrow$ ). In contrast, increasing lung cancer mortality rates reduced $\mathrm{LE}_{40-84}$ in women in many very high HDI populations (an average of nearly 0.1 years loss), with the greatest loss observed in the Netherlands and Hungary ( 0.3 and 0.2 years, respectively). Although lung cancer had little impact on the change in $\mathrm{LE}_{40-84}$ among women in medium and high HDI populations (table $3 \Downarrow$ ), decreasing lung cancer mortality rates led to gains of 0.3 and 0.2 years among men in Azerbaijan and Georgia, respectively.

Among women, declines in breast cancer mortality rates had a positive effect on longevity in almost all populations in the very high HDI category (on average 0.1 years gain), particularly in the United Kingdom, with an $\mathrm{LE}_{40-84}$ gain of 0.3 years in 1981-2010. In contrast, there was a reduction in $\mathrm{LE}_{40-84}$ in women (up to 0.1 year) owing to increasing breast cancer mortality rates in several Asian, South American, and former Soviet Union populations indexed as medium and high HDI, including Kuwait,
Ukraine, and El Salvador. Similarly, prostate cancer among men contributed a slight reduction in $\mathrm{LE}_{40-84}$ in medium and high HDI populations, notably in Cuba (almost 0.1 years); conversely, prostate cancer contributed to a gain in male $\mathrm{LE}_{40-84}$ in several very high HDI populations, with the largest gain in Switzerland (0.2 years).

Finally, the uniform declines in mortality rates from stomach cancer led to universal gains in $\mathrm{LE}_{40-84}$, other than for Guatemala and El Salvador. The largest gain was observed in the Republic of Korea ( 0.6 and 0.4 years in men and women, respectively). Results were less consistent for colorectal cancer. In most populations, particularly those at very high HDI levels, declining mortality rates from colorectal cancer resulted in gains in $\mathrm{LE}_{40-84}$. The gain was equivalent to 0.2 years among Australian men; however, several former Soviet Union populations experienced a reduction in $\mathrm{LE}_{40-84}$ as a result of increasing mortality rates from colorectal cancer.

\section{Discussion}

In this study, we quantified the extent to which cancer has contributed to changes in life expectancy in ages $40-84\left(\mathrm{LE}_{40-84}\right)$ over the past three decades, benchmarking gains and losses in longevity with those due to changing mortality rates from cardiovascular disease (CVD). Overall, in 52 populations with long term vital registration systems, declines in cancer mortality rates between 1981 and 2010 have independently contributed to an improvement in longevity and the magnitude of the improvement over time due to decline in cancer mortality rates is inextricably linked with a population's level of development (Human Development Index (HDI)). Specifically, declines in cancer mortality rates among men contributed about one fifth of the overall years gained in ages 40-84, or an average of 0.8 years, in very high HDI populations, in contrast with the one seventh overall gain, or 0.2 years, in medium and high HDI populations. Inequalities in life year gains among women were of a lesser magnitude but still apparent $(0.5$ and 0.2 years gained, respectively). An increasing impact of declines in cancer mortality rates on longevity can be anticipated, particularly in higher resourced settings, given the substantial lag between the introduction of effective programs of prevention, early detection and treatment, and corresponding reductions in the cancer burden.

Evidently, declines in mortality rates were greatest for CVD, particularly in very high and in medium and high HDI populations, with gains in $\mathrm{LE}_{40-84}$ of 1.7 years in women and up to 2.3 years in men; results consistent with previous studies. ${ }^{2324}$ Over the past three decades there have been major advances in the control of cardiovascular risk factors (smoking, blood pressure, and cholesterol levels) as well as improvements in medical care in many high HDI countries. ${ }^{25}$ The restricted effect of reductions in cancer mortality rates in advancing longevity partly results from the considerable heterogeneity in underlying risk factors and a longer latency period and interval after which benefits from preventive interventions can be observed. ${ }^{26}$ For example, among smokers, the risk of death from CVD decreases rapidly after cessation and reaches that of non-smokers within five to 10 years, in contrast with cancer where the impact can take three or more decades. ${ }^{25-28}$ In addition, treatment for CVD is highly effective, with almost half of the decline in CVD deaths in the USA in 1980-2000 attributed to improved treatment, ${ }^{29}$ whereas for cancer, despite many breakthroughs, effective treatments for several major sites are still lacking, with over $80 \%$ of patients with lung cancer or liver cancer dying within five years after diagnosis. ${ }^{30}$ 
Our results also show noticeable population and sex differences in the extent of changes in longevity over time, particularly for lung cancer, presumably driven by the distinct population specific and sex specific historical trends of the tobacco epidemic. ${ }^{31-34}$ The most remarkable contrast was observed in the Netherlands: Dutch men experienced the greatest gain in $\mathrm{LE}_{40-84}$ as a result of declining lung cancer mortality rates, whereas Dutch women experienced the greatest loss in $\mathrm{LE}_{40-84}$, largely as a result of increasing lung cancer mortality rates. Similar patterns are widely observed in other very high HDI populations where smoking rates have been increasing in women compared with men, in part linked to their increasing empowerment. ${ }^{32-36}$ Sustained upward trends in smoking rates in women and consequent loss in longevity related to cancer have yet to be seen in transitioning populations, partly because of continuing sex inequalities. ${ }^{32} 37$

\section{Strengths and limitations of this study}

The methodology we present enables the estimation of the independent contribution of change in cancer mortality rates to changes in life expectancy over time by simultaneously accounting for changes in other causes of death. Our results are thus not confounded by non-cancer related deaths, as was shown in a previous US study. ${ }^{22}$ The interplay between cancers and other causes of death is complex, however, particularly in populations undergoing major social and economic transitions. Taking into account other causes of deaths is therefore a necessity in order to estimate the effectiveness of cancer control programs.

Our study has important limitations. Firstly, only a small proportion of eligible populations had the requisite data covering the calendar period under study; as a result only a few populations included in this study were indexed as having medium HDI (eg, Guatemala). ${ }^{19}$ Since most low HDI and many medium HDI populations (largely overlap with low and middle income countries) are at the beginning of the epidemiological transition, our analysis of medium to very high HDI populations compares populations for which such transitions range from ongoing to fully completed. Nevertheless, the implications of our findings are of great relevance to populations in low and middle income countries, as they are expected to follow the path of epidemiological transition undergone in higher resourced settings.

Secondly, while other macroeconomic indicators of healthcare, income, or inequality - for example, the availability of universal healthcare or the distribution of wealth at the global or regional level could undoubtedly have been used, we chose HDI as a standardized measure that asserts the fact that human choices are important in assessing societal gains, in addition to economic development.

Thirdly, the information on death counts and the underlying cause of death tends to be less accurate in less resourced populations, leading to a corresponding lack of validity of recorded mortality. We have, however, included only populations appraised as having medium and higher levels of data quality, and thus our results are unlikely to be substantively affected by data quality issues. ${ }^{16} \mathrm{~A}$ strict inclusion criterion based on data quality will also minimize inconsistent results that might arise from changes in ICD coding overtime. ${ }^{16}{ }^{38} \mathrm{In}$ addition, visual examinations of age-standardized mortality rates by cause do not show notable discontinuities. Moreover, we only included the most common causes of death, which tend to be associated with a relatively low probability of being misclassified in the studied populations and of being inconsistent as a result of variations in coding practice across countries. ${ }^{16-40}$

Lastly, owing to a lack of information on risk factors and national cancer control practices, we did not quantify the variability in life years gained or lost due to specific factors over time; this partitioning of changes in life expectancy according to changing lifestyles and the specific impact of prevention and early detection and treatment interventions remains an important issue to investigate in future studies.

\section{Implications of findings}

The past three decades have been marked by several triumphs in cancer control, which are clearly reflected in our results. For example, the increase in $\mathrm{LE}_{40-84}$ among men can be considered partly the result of corresponding declines in lung cancer mortality rates linked to improved tobacco control measures. ${ }^{41}$ The WHO's Framework Convention on Tobacco Control has now been ratified internationally, but the comprehensive tobacco control programs proposed through the MPOWER policy package have been only marginally adopted in low and middle income countries. ${ }^{33}$ Tax hikes, an intervention that has been shown to be highly effective in reducing smoking prevalence, has remained the least achieved measure, with only one low income country (Madagascar) introducing a tax of at least $75 \%$ of the tobacco retail price (versus 20 countries in high income settings) in $2013 .{ }^{33}$ The potential health impact of such an intervention is tremendous; a price increase of $70 \%$ could potentially lead to the avoidance of 110 million deaths globally, including 25 million from cancer. ${ }^{34}$ Further strengthening of sustainable tobacco control policies is fundamental, not only in low and middle income countries but also in high income settings. The uptake of cigarette smoking and other new forms of tobacco products among young people in many high income countries poses a great threat to future increases in life expectancy. ${ }^{42}{ }^{43}$ Effective sex specific tobacco control strategies are also particularly needed to curb the burdens from non-communicable diseases in low and middle income countries where current smoking rates in women are low but expected to increase as women are being progressively empowered in these populations. $^{3344}$

Gains in life expectancy from a reduction of stomach cancer mortality rates links socioeconomic advancement to successes in combating infectious diseases through both "unplanned" prevention as well as treatment. ${ }^{45}{ }^{46}$ Our analysis shows that very high HDI populations have benefited from larger declines compared with medium and high HDI populations. Notable progress in reducing rates of stomach cancer has been observed in Asia, particularly in Japan and the Republic of Korea, where national programs of early diagnosis and treatment co-exist and declines in exposure to Helicobacter pylori infection have been observed. ${ }^{47}$ Other major infection associated cancers, including cervical and liver cancer are largely preventable or treatable. The implementation of affordable yet effective essential packages of interventions included in the cancer volume of Disease Control Priorities-3 aims to ensure universal reduction of premature deaths from cancer. ${ }^{48-50}$ If fully implemented, this package will only induce an additional annual expenditure per capita of approximately $\$ 5.7$ ( $£ 4.4 ; € 5.1$ ) in upper middle income countries and $\$ 1.7$ in low income and lower middle income countries. ${ }^{51}$

In most of the very high HDI populations, the progress seen in terms of reductions in mortality rates from breast, prostate, and colorectal cancer can be related to a broad spectrum of cancer control interventions, including early detection, improved 
diagnosis, and better access to effective treatment. ${ }^{30}$ In many such populations the health focus has shifted from increased longevity in itself to improved quality of life at older ages, to increase healthy life years, as quality of life can be negatively affected after a cancer diagnosis. ${ }^{52}$ An expansion of such interventions to low and middle income countries remains a great challenge, but could partly be tackled through adoption of essential medicine packages. ${ }^{53-55}$ The gap to bridge remains large, however, with, for example, surgery, central to treatment for solid tumors, available for $85 \%$ of the population in highly resourced settings compared with $1 \%$ in low resourced settings. ${ }^{56}$ Furthermore, radiotherapy, an essential treatment modality for more than half of all patients with cancer, is minimal or absent in these settings, with as few as one radiotherapy unit per five million people estimated. ${ }^{57}$ Major improvements in global and domestic financing to ensure better access to cancer screening, surgery, essential medicines, and radiotherapy services, along with an expansion of universal health coverage to protect against catastrophic health expenditures are critical for countries to respond to the increasing burden of cancer. ${ }^{57}$ Placing the emphasis on prevention strategies would be an especially attractive option for low and middle income countries, as such strategies are more cost effective and will simultaneously reduce the loss in life expectancy and years spent with disability due to cancer. ${ }^{51}$ Additionally, cancer control policies should also take inequality within populations into account, as the disadvantaged subgroups often face disproportionally more barriers and greater economic burden to reap the benefits of such policies. ${ }^{58} 59$

\section{Conclusions}

Many populations, particularly those with greater resources, have benefited from a rising life expectancy as a direct result of a reduction in mortality rates from cancer since 1981 . These gains are attributable to advancements in cancer control strategies over the past 30 years. However, in many lower resourced populations such gains have yet to be seen. Meeting the targets of the WHO Global Action Plan for non-communicable diseases and achieving the sustainable development goal to reduce premature mortality from non-communicable diseases by one third by 2030 are priorities for WHO member states. ${ }^{1-61}$ While significant progress in CVD control has been made, additional resources need to be urgently allocated to the development and implementation of effective, affordable, feasible, and sustainable cancer control measures in countries undergoing socioeconomic and health transitions.

We thank Jacques Ferlay for the provision of cancer specific mortality data, Mathieu Laversanne for assistance in graphical design of the study results, and Miranda Fidler for assistance with reading earlier versions of this manuscript.

Contributors: BC contributed to data collection, study design, data analysis, and interpretation of the results, and wrote the first draft of the paper. IS and FB contributed to the study design, interpretation of the results, and drafting of the paper. HBS and SS contributed to the study design, interpretation of the results, and finalizing the paper. OG contributed to interpretation of the results and finalizing the paper. IS is the study guarantor and had final responsibility for the decision to submit for publication. All authors read and approved the final version of the paper.

Funding: None.

Competing interests: All authors have completed the ICMJE uniform disclosure form at www.icmje.org/coi_disclosure.pdf and declare: no support from any organization for the submitted work; no financial relationships with any organizations that might have an interest in the submitted work in the previous three years; no other relationships or activities that could appear to have influenced the submitted work.

\section{Ethical approval: Not required.}

Data sharing: The full dataset is available online (www.who.int/healthinfo/ mortality_data/en/) and can be requested from the corresponding author. Transparency: The lead author (BC) affirms that the manuscript is an honest, accurate, and transparent account of the study being reported; that no important aspects of the study have been omitted; and that any discrepancies from the study as planned have been explained.

1 Bray F, Jemal A, Grey N, Ferlay J, Forman D. Global cancer transitions according to the Human Development Index (2008-2030): a population-based study. Lancet Oncol 2012:13:790-801. doi:10.1016/S1470-2045(12)70211-5pmid:22658655.

2 Torre LA, Bray F, Siegel RL, Ferlay J, Lortet-Tieulent J, Jemal A. Global cancer statistics, 2012. CA Cancer J Clin 2015;65:87-108. doi:10.3322/caac.21262pmid:25651787.

3 Townsend N, Wilson L, Bhatnagar P, Wickramasinghe K, Rayner M, Nichols M. Cardiovascular disease in Europe: epidemiological update 2016. Eur Heart $J$ 2016;37:3232-45. doi:10.1093/eurheartj/ehw334. pmid:27523477.

4 International Agency for Research on Cancer (IARC). GLOBOCAN 2012: Estimated Cancer Incidence, Mortality and Prevalence Worldwide in 2012. Globocan 2012;2012:3-6.

5 Caldwell JC. Toward A Restatement of Demographic Transition Theory. Pop Develop Rev 1976;2:321-66.

6 Kanavos P. The rising burden of cancer in the developing world. Ann Oncol 2006;17(Suppl 8):i15, i23. doi:10.1093/annonc/mdl983pmid:16801335.

7 Omran AR. The epidemiologic transition. A theory of the epidemiology of population change. Milbank Mem Fund Q 1971:49:509-38. doi:10.2307/3349375pmid:5155251.

8 DeSantis CE, Bray F, Ferlay J, Lortet-Tieulent J, Anderson BO, Jemal A. International Variation in Female Breast Cancer Incidence and Mortality Rates. Cancer Epidemiol Biomarkers Prev 2015;24:1495-506. doi:10.1158/1055-9965.EPI-15-0535pmid:26359465.

9 Torre LA, Siegel RL, Ward EM, Jemal A. International variation in lung cancer mortality rates and trends among women. Cancer Epidemiol Biomarkers Prev 2014;23:1025-36. doi:10.1158/1055-9965.EPI-13-1220pmid:24836468.

10 Ferro A, Peleteiro B, Malvezzi M, et al. Worldwide trends in gastric cancer mortality (1980-2011), with predictions to 2015, and incidence by subtype. Eur J Cancer 2014;50:1330-44. doi:10.1016/j.ejca.2014.01.029pmid:24650579.

11 Siegel R, Desantis C, Jemal A. Colorectal cancer statistics, 2014. CA Cancer J Clin 2014;64:104-17. doi:10.3322/caac.21220pmid:24639052.

12 Center MM, Jemal A, Lortet-Tieulent J, et al. International variation in prostate cancer incidence and mortality rates. Eur Urol 2012;61:1079-92. doi:10.1016/j.eururo.2012.02. 054pmid:22424666.

13 World Bank. World Bank: Country and Lending Groups Data. World Bank Data Website, 2016.

14 World Health Organization. WHO Mortality Database. 2014. http://www.who.int/healthinfo/ statistics/mortality rawdata/en/.

15 United Nations. World Population Prospects - Population Division - United Nations. 2015

16 Mathers CD, Fat DM, Inoue M, Rao C, Lopez AD. Counting the dead and what they died from: an assessment of the global status of cause of death data. Bull World Health Organ 2005;83:171-7.pmid:15798840.

17 Department of Health Statistics and Information Systems, WHO. WHO methods and data sources for global causes of death, 2000-2015. 2016.

18 Ferlay J, Soerjomataram I, Dikshit R, et al. Cancer incidence and mortality worldwide: sources, methods and major patterns in GLOBOCAN 2012. Int $J$ Cancer 2015;136:E359-86. doi:10.1002/ijc.29210pmid:25220842.

19 United Nations Development Programme. Human development report. DOI, 2015.

20 Preston SH, Heuveline P, Guillot M. Demography: Measuring and Modeling Population Processes. Wiley, 2000, 10.2307/1535065.

21 Beltrán-Sánchez H, Preston SH, Canudas-Romo V. An integrated approach to cause-of-death analysis: cause-deleted life tables and decompositions of life expectancy. Demogr Res 2008;19:1323-50. doi:10.4054/DemRes.2008.19.35pmid:20165568.

22 Soneji S, Beltrán-Sánchez H, Sox HC. Assessing progress in reducing the burden of cancer mortality, 1985-2005. J Clin Oncol 2014;32:444-8. doi:10.1200/JCO.2013.50. 8952pmid:24419122.

23 Mathers CD, Stevens GA, Boerma T, White RA, Tobias MI. Causes of international increases in older age life expectancy. Lancet 2015;385:540-8. doi:10.1016/S0140-6736( 14)60569-9pmid:25468166.

24 Klenk J, Keil U, Jaensch A, Christiansen MC, Nagel G. Changes in life expectancy 1950-2010: contributions from age- and disease-specific mortality in selected countries. Popul Health Metr 2016:14:20. doi:10.1186/s12963-016-0089-xpmid:27222639.

25 Ezzati M, Obermeyer Z, Tzoulaki I, Mayosi BM, Elliott P, Leon DA. Contributions of risk factors and medical care to cardiovascular mortality trends. Nat Rev Cardiol 2015;12:508-30. doi:10.1038/nrcardio.2015.82pmid:26076950.

26 Wild CP, Bray F, Forman D, Franceschi S, Sankaranarayanan R, Straif K. Cancer in the 25×25 non-communicable disease targets. Lancet 2014;384:1502-3. doi:10.1016/S01406736(14)61918-8pmid:25390574.

27 Kontis V, Mathers CD, Rehm J, et al. Contribution of six risk factors to achieving the $25 \times 25$ non-communicable disease mortality reduction target: a modelling study. Lancet 2014;384:427-37. doi:10.1016/S0140-6736(14)60616-4pmid:24797573.

28 Oza S, Thun MJ, Henley SJ, Lopez AD, Ezzati M. How many deaths are attributable to smoking in the United States? Comparison of methods for estimating smoking-attributable mortality when smoking prevalence changes. Prev Med 2011;52:428-33. doi:10.1016/j. ypmed.2011.04.007pmid:21530575.

29 Ford ES, Ajani UA, Croft JB, et al. Explaining the decrease in U.S. deaths from coronary disease, 1980-2000. N Engl J Med 2007;356:2388-98. doi:10.1056/NEJMsa053935. pmid: 17554120.

30 Allemani C, Weir HK, Carreira H, et al. CONCORD Working Group. Global surveillance of cancer survival 1995-2009: analysis of individual data for 25,676,887 patients from 279 population-based registries in 67 countries (CONCORD-2). Lancet 2015;385:977-1010. doi:10.1016/S0140-6736(14)62038-9pmid:25467588. 


\section{What is already known on this topic}

Cancer is becoming a leading cause of morbidity and mortality worldwide, as a result of epidemiological transition

The incidence rates of many cancers are increasing or expected to increase in many low and middle income countries, in part due to the uptake of unhealthy lifestyles

Mortality rates from cancers and cardiovascular disease (CVD) are declining in most highly developed countries, but are still rising or at best stabilizing in transitioning countries, thus requiring a global assessment of these diseases as barriers to attaining old age

\section{What this study adds}

Declining mortality from CVD was responsible for about half of the life expectancy gains in ages 40-84 in 1981-2010, whereas the contribution from cancer is smaller (up to one fifth) and varied depending on a country's level of development

Among all cancer related life expectancy gains in ages $40-84$, the greatest were achieved through reductions in mortality rates from lung cancer and breast cancer in women

31 Waldron I. Patterns and causes of gender differences in smoking. Soc Sci Med 1991;32:989-1005. doi:10.1016/0277-9536(91)90157-8. pmid:2047903.

32 Pampel FC. Global Patterns and Determinants of Sex Differences in Smoking. Int J Comp Sociol 2006;47:466-87. doi:10.1177/0020715206070267. pmid:21874066.

33 World Health Organization. WHO Report on the Global Tobacco Epidemic. WHO Rep Glob Tob Epidemic 2013;5:106.

34 Jha P. Avoidable global cancer deaths and total deaths from smoking. Nat Rev Cancer 2009;9:655-64. doi:10.1038/nrc2703pmid:19693096.

35 Graham H. Smoking prevalence among women in the European community 1950-1990. Soc Sci Med 1996;43:243-54. doi:10.1016/0277-9536(95)00369-X. pmid:8844928.

36 Schaap MM, Kunst AE, Leinsalu M, et al. Female ever-smoking, education, emancipation and economic development in 19 European countries. Soc Sci Med 2009;68:1271-8. doi: 10.1016/j.socscimed.2009.01.007. pmid:19195749.

37 Waldron I, Bratelli G, Carriker L, Sung WC, Vogeli C, Waldman E. Gender differences in tobacco use in Africa, Asia, the Pacific, and Latin America. Soc Sci Med 1988;27:1269-75. doi:10.1016/0277-9536(88)90357-7. pmid:3206258.

38 Janssen F, Kunst AE. ICD coding changes and discontinuities in trends in cause-specific mortality in six European countries, 1950-99 I. Bull World Health Organ 2004. doi:/S0042-96862004001200006.

39 Danilova I, Shkolnikov VM, Jdanov DA, Meslé F, Vallin J. Identifying potential differences in cause-of-death coding practices across Russian regions. Popul Health Metr 2016;14:8. doi:10.1186/s12963-016-0078-0. pmid:27006644.

40 Meslé F, Vallin J. Reconstructing series of deaths by cause with constant definitions. In: Mortality and causes of death in 20th-century Ukraine. 2012:131-52.

41 IARC Working Group on the Evaluation of Carcinogenic Risks to Humans. Tobacco smoke and involuntary smoking. IARC Monogr Eval Carcinog Risks Hum 2004;83:1-1438.pmid: 15285078.

42 OFDT. Drogues et addictions, données essentielles. 2013 doi:10.3917/lcd.056.0143.

43 Singh T, Arrazola RA, Corey CG, et al. Tobacco Use Among Middle and High School Students--United States, 2011-2015. MMWR Morb Mortal Wkly Rep 2016;65:361-7. doi: 10.15585/mmwr.mm6514a1. pmid:27077789.

44 Samet J, Yoo S. Women and the Tobacco Epidemic: Challenges for the 21st Century. World Heal Organ, 2001

45 Yamaguchi N, Kakizoe T. Synergistic interaction between Helicobacter pylori gastritis and diet in gastric cancer. Lancet Oncol 2001;2:88-94. doi:10.1016/S1470-2045(00)002254pmid:11905800.

46 Howson CP, Hiyama T, Wynder EL. The decline in gastric cancer: epidemiology of an unplanned triumph. Epidemiol Rev 1986;8:1-27. doi:10.1093/oxfordjournals.epirev. a036288pmid:3533579.

47 Leung WK, Wu MS, Kakugawa Y, et al. Asia Pacific Working Group on Gastric Cancer. Screening for gastric cancer in Asia: current evidence and practice. Lancet Oncol 2008:9:279-87. doi:10.1016/S1470-2045(08)70072-Xpmid:18308253

48 Jamison DT, Summers LH, Alleyne G, et al. Global health 2035: a world converging within a generation. Lancet 2013;382:1898-955. doi:10.1016/S0140-6736(13)62105-4pmid: 24309475
49 de Martel C, Ferlay J, Franceschi S, et al. Global burden of cancers attributable to infections in 2008: a review and synthetic analysis. Lancet Oncol 2012;13:607-15. do: 10.1016/S1470-2045(12)70137-7pmid:22575588.

50 Gelband H, Jha P, Sankaranarayanan R, Horton S. Disease Control Priorities, Third Edition (Volume 3): Cancer. Dis Control Priorities, Third Ed (Volume 3) 2015. doi:10.1596/ 978-1-4648-0349-9.

51 Gelband H, Sankaranarayanan R, Gauvreau CL, et al. Costs, affordability, and feasibility of an essential package of cancer control interventions in low-income and middle-income countries: Key messages from Disease Control Priorities, 3rd edition. Lancet 2016;387:2133-44

52 Soerjomataram I, Lortet-Tieulent J, Parkin DM, et al. Global burden of cancer in 2008: a systematic analysis of disability-adjusted life-years in 12 world regions. Lancet 2012;380:1840-50. doi:10.1016/S0140-6736(12)60919-2pmid:23079588

53 World Health Organization. 19th WHO Model List of Essential Medicines. 2015;1-43.

54 Farmer P, Frenk J, Knaul FM, et al. Expansion of cancer care and control in countries of low and middle income: a call to action. Lancet 2010;376:1186-93. doi:10.1016/S01406736(10)61152-Xpmid:20709386.

55 Ginsburg O, Bray F, Coleman MP, et al. The global burden of women's cancers: a grand challenge in global health. Lancet 2017;389:847-60. doi:10.1016/S0140-6736(16)313927. pmid:27814965.

56 Alkire BC, Raykar NP, Shrime MG, et al. Global access to surgical care: a modelling study. Lancet Glob Health 2015;3:e316-23. doi:10.1016/S2214-109X(15)70115-4pmid: 25926087.

57 Atun R, Jaffray DA, Barton MB, et al. Expanding global access to radiotherapy. Lancet Oncol 2015;16:1153-86. doi:10.1016/S1470-2045(15)00222-3pmid:26419354.

58 Thomson GW. Wilson N a, O'Dea D, Reid PJ, Howden-Chapman P. Tobacco spending and children in low income households. Tob Control 2002;11:372-5. doi:10.1136/tc.11.4. 372.

59 Strasser-Weippl K, Chavarri-Guerra Y, Villarreal-Garza C, et al. Progress and remaining challenges for cancer control in Latin America and the Caribbean. Lancet Oncol 2015;16:1405-38. doi:10.1016/S1470-2045(15)00218-1. pmid:26522157.

60 World Health Organization. Global Action Plan for the prevention and control of NCDs 2013-2020. http://www.who.int/nmh/events/ncd_action_plan/en/

61 Maurice J. UN set to change the world with new development goals. Lancet 2015;386:1121-4. doi:10.1016/S0140-6736(15)00251-2pmid:26461891.

\section{Accepted: 24052017}

Published by the BMJ Publishing Group Limited. For permission to use (where not already granted under a licence) please go to http://group.bmj.com/group/rights-licensing permissions

This is an Open Access article distributed in accordance with the Creative Commons Attribution Non Commercial (CC BY-NC 4.0) license, which permits others to distribute, remix, adapt, build upon this work non-commercially, and license their derivative works on different terms, provided the original work is properly cited and the use is non-commercial. See: http://creativecommons.org/licenses/by-nc/4.0/. 


\section{Tables}

Table 1| Life expectancy and mortality rates for ages 40-84 years in 1981-85 and 2006-10 by Human Development Index (HDI)

\begin{tabular}{|c|c|c|c|c|c|c|c|c|c|c|c|c|}
\hline \multirow{3}{*}{$\begin{array}{l}\text { Variables in } \\
\text { ages } 40-84 \\
\text { years }\end{array}$} & \multicolumn{6}{|c|}{ Men } & \multicolumn{6}{|c|}{ Women } \\
\hline & \multicolumn{3}{|c|}{ Very high HDI } & \multicolumn{3}{|c|}{ Medium and high HDI } & \multicolumn{3}{|c|}{ Very high HDI } & \multicolumn{3}{|c|}{ Medium and high HDI } \\
\hline & $1981-85$ & 2006-10 & Change (\%) & $1981-85$ & 2006-10 & Change (\%) & $1981-85$ & 2006-10 & Change (\%) & $1981-85$ & 2006-10 & Change (\%) \\
\hline Life expectancy & 32.4 & 36.1 & $3.7(11.6 \%)$ & 31.5 & 32.6 & $1.1(3.5 \%)$ & 37.1 & 39.6 & $2.5(6.7 \%)$ & 35.8 & 37.2 & $1.4(3.9 \%)$ \\
\hline \multicolumn{13}{|l|}{$\begin{array}{l}\text { Age-standardized } \\
\text { mortality rates by } \\
\text { cause: }\end{array}$} \\
\hline All causes & 1948.5 & 1217.5 & $\begin{array}{c}-730.9 \\
(-37.5 \%)\end{array}$ & 2134.1 & 1922.7 & $\begin{array}{l}-211.4 \\
(-9.9 \%)\end{array}$ & 1039.1 & 652.1 & $\begin{array}{c}-387 \\
(-37.2 \%)\end{array}$ & 1260.7 & 1040.3 & $\begin{array}{c}-220.4 \\
(-17.5 \%)\end{array}$ \\
\hline $\begin{array}{l}\text { Cardiovascular } \\
\text { diseases }\end{array}$ & 878.0 & 414.8 & $\begin{array}{c}-463.2 \\
(-52.8 \%)\end{array}$ & 956.9 & 853.1 & $\begin{array}{c}-103.8 \\
(-10.8 \%)\end{array}$ & 473.3 & 203.8 & $\begin{array}{l}-269.5 \\
(-56.9 \%)\end{array}$ & 618.2 & 480.0 & $\begin{array}{c}-138.2 \\
(-22.4 \%)\end{array}$ \\
\hline All cancers & 490.3 & 392.5 & $\begin{array}{c}-97.7 \\
(-19.9 \%) \\
\end{array}$ & 341.1 & 327.4 & $-13.7(-4.0 \%)$ & 285.3 & 242.1 & $\begin{array}{c}-43.3 \\
(-15.2 \%) \\
\end{array}$ & 219.2 & 208.1 & $-11.1(-5.1 \%)$ \\
\hline Lung cancer & 148.5 & 108.9 & $\begin{array}{c}-39.5 \\
(-26.6 \%)\end{array}$ & 86.9 & 75.2 & $\begin{array}{c}-11.7 \\
(-13.5 \%) \\
\end{array}$ & 29.5 & 41.3 & $11.8(39.9 \%)$ & 15.2 & 17.0 & $1.8(11.8 \%)$ \\
\hline Stomach cancer & 59.0 & 25.6 & $\begin{array}{c}-33.4 \\
(-56.5 \%) \\
\end{array}$ & 70.2 & 39.5 & $\begin{array}{c}-30.7 \\
(-43.7 \%) \\
\end{array}$ & 26.0 & 10.7 & $\begin{array}{c}-15.3 \\
(-58.8 \%) \\
\end{array}$ & 32.7 & 18.0 & $\begin{array}{c}-14.7 \\
(-45.0 \%) \\
\end{array}$ \\
\hline $\begin{array}{l}\text { Colorectal } \\
\text { cancer }\end{array}$ & 46.2 & 43.7 & $-2.5(-5.4 \%)$ & 22.2 & 29.1 & $6.9(31.1 \%)$ & 33.6 & 26.3 & $-7.3(-21.7 \%)$ & 18.2 & 19.8 & $1.6(8.8 \%)$ \\
\hline Prostate cancer & 32.4 & 30.3 & $-2.1(-6.6 \%)$ & 20.2 & 31.1 & $10.9(54.0 \%)$ & - & - & - & - & - & - \\
\hline Breast cancer & - & - & - & - & - & - & 52.7 & 43.4 & $-9.3(-17.6 \%)$ & 29.8 & 35.9 & $6.1(20.5 \%)$ \\
\hline All other causes & 580.2 & 410.2 & $\begin{array}{l}-170.0 \\
(-29.3 \%)\end{array}$ & 836.1 & 742.2 & $\begin{array}{c}-93.9 \\
(-11.2 \%)\end{array}$ & 280.5 & 206.1 & $\begin{array}{c}-74.3 \\
(-26.5 \%)\end{array}$ & 423.3 & 352.2 & $\begin{array}{c}-71.1 \\
(-16.8 \%)\end{array}$ \\
\hline
\end{tabular}

Life Expectancy corresponds to ages 40-84, expressed in years.

Age-standardized mortality rates (ASMR), using world standard population, in ages 40-84 per 100000 . 
Table 2| Proportion of deaths from specific deaths in all deaths for ages 40-84 years in 1981-85 and 2006-10 by Human Development Index (HDI). Values are percentages

\begin{tabular}{|c|c|c|c|c|c|c|c|c|c|c|c|c|}
\hline \multirow[b]{3}{*}{ Causes of death } & \multicolumn{6}{|c|}{ Men } & \multicolumn{6}{|c|}{ Women } \\
\hline & \multicolumn{3}{|c|}{ Very high HDI } & \multicolumn{3}{|c|}{ Medium and high HDI } & \multicolumn{3}{|c|}{ Very high HDI } & \multicolumn{3}{|c|}{ Medium and high HDI } \\
\hline & $1981-85$ & 2006-10 & Change & $1981-85$ & 2006-10 & Change & $1981-85$ & 2006-10 & Change & $1981-85$ & 2006-10 & Change \\
\hline CVD & 45.1 & 34.1 & -11.0 & 44.8 & 44.4 & -0.5 & 45.5 & 31.3 & -14.3 & 49.0 & 46.1 & -2.9 \\
\hline All cancers & 25.2 & 32.2 & 7.1 & 16.0 & 17.0 & 1.0 & 27.5 & 37.1 & 9.7 & 17.4 & 20.0 & 2.6 \\
\hline Lung cancer & 7.6 & 8.9 & 1.3 & 4.1 & 3.9 & -0.2 & 2.8 & 6.3 & 3.5 & 1.2 & 1.6 & 0.4 \\
\hline Stomach cancer & 3.0 & 2.1 & -0.9 & 3.3 & 2.1 & -1.2 & 2.5 & 1.6 & -0.9 & 2.6 & 1.7 & -0.9 \\
\hline Colorectal cancer & 2.4 & 3.6 & 1.2 & 1.0 & 1.5 & 0.5 & 3.2 & 4.0 & 0.8 & 1.4 & 1.9 & 0.5 \\
\hline Prostate cancer & 1.7 & 2.5 & 0.8 & 0.9 & 1.6 & 0.7 & - & - & - & - & - & - \\
\hline Breast cancer & - & - & - & - & - & - & 5.1 & 6.7 & 1.6 & 2.4 & 3.5 & 1.1 \\
\hline All other causes & 29.8 & 33.7 & 3.9 & 39.2 & 38.6 & -0.6 & 27.0 & 31.6 & 4.6 & 33.6 & 33.9 & 0.3 \\
\hline
\end{tabular}

CVD=cardiovascular disease.

Proportions estimated by dividing Age-standardized mortality rates (ASMR) from indexed disease by ASMR from all causes for ages $40-84$. 
Table 3| Average contribution (in years) of each cause of death to change in life expectancy in ages 40-84 years, between 1981-85 and 2006-10

\begin{tabular}{|c|c|c|c|c|c|c|c|c|}
\hline \multirow[b]{3}{*}{ Causes of death } & \multicolumn{4}{|c|}{ Men } & \multicolumn{4}{|c|}{ Women } \\
\hline & \multicolumn{2}{|c|}{ Very high HDI } & \multicolumn{2}{|c|}{ Medium and high HDI } & \multicolumn{2}{|c|}{ Very high HDI } & \multicolumn{2}{|c|}{ Medium and high HDI } \\
\hline & Absolute & $\%$ & Absolute & $\%$ & Absolute & $\%$ & Absolute & $\%$ \\
\hline Cardiovascular diseases & 2.3 & 60.9 & 0.5 & 43.2 & 1.7 & 67.3 & 0.8 & 56.8 \\
\hline All cancers & 0.8 & 22.6 & 0.2 & 14.4 & 0.5 & 20.5 & 0.2 & 11.1 \\
\hline Lung cancer & 0.3 & 7.6 & 0.1 & 7.0 & 0.0 & -1.7 & 0.0 & 0.3 \\
\hline Stomach cancer & 0.2 & 5.8 & 0.1 & 13.3 & 0.1 & 5.5 & 0.1 & 7.3 \\
\hline Colorectal cancer & 0.1 & 2.0 & 0.0 & -1.1 & 0.1 & 3.5 & 0.0 & 0.5 \\
\hline Prostate cancer & 0.1 & 1.8 & 0.0 & -2.3 & - & - & - & - \\
\hline Breast cancer & - & - & - & - & 0.1 & 4.5 & 0.0 & -1.3 \\
\hline All other cancers & 0.2 & 5.5 & 0.0 & -2.5 & 0.2 & 8.7 & 0.1 & 4.2 \\
\hline All other causes & 0.6 & 16.5 & 0.4 & 34.0 & 0.3 & 12.2 & 0.4 & 26.5 \\
\hline Overall change & 3.7 & 100.0 & 1.1 & 100.0 & 2.5 & 100.0 & 1.4 & 100.0 \\
\hline
\end{tabular}

HDI=Human Development Index. 


\section{Figures}

\section{冈}

Fig 1 Changes in age standardized mortality rates in ages 40-84 years between 1981-85 and 2006-10 due to cardiovascular disease (CVD), all cancers, and all other causes of death in men and women combined. HDI=Human Development Index

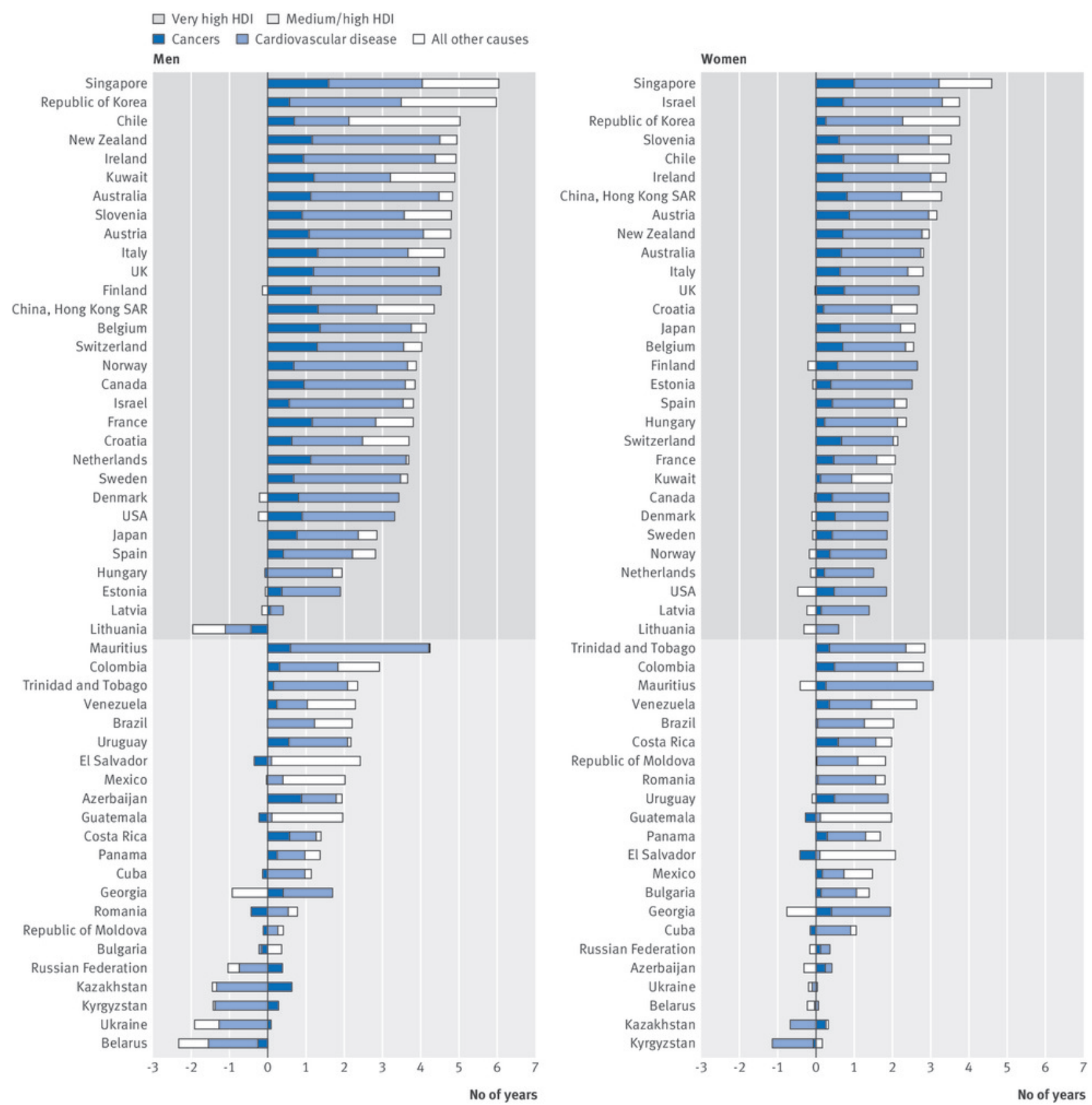

Fig 2 Changes in overall life expectancy in ages 40-84 years between 1981-85 and 2006-10 due to cardiovascular disease (CVD), all cancers, and all other causes of death in men and women. HDI=Human Development Index 

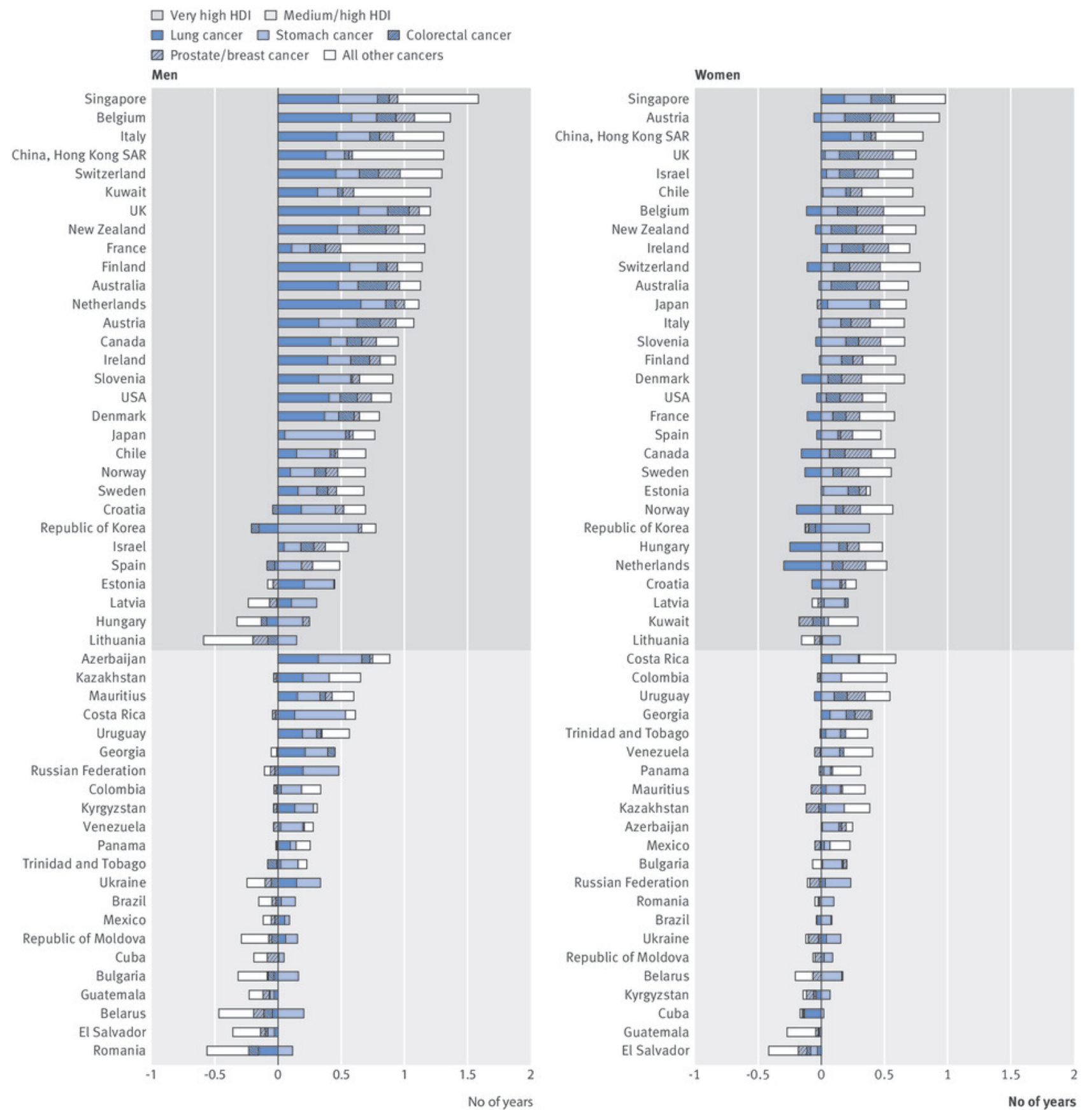

Fig 3 Changes in overall life expectancy in ages 40-84 years between 1981-85 and 2006-10 due to specific major cancer types in men and women. $\mathrm{HDI}=\mathrm{Human}$ Development Index 